\title{
Analysis of properties of optical carriers after long-term storage
}

\author{
V.V. Petrov ${ }^{1}$, A.A. Kryuchyn ${ }^{1}$, I.V. Gorbov ${ }^{1}$, I.O. Kossko ${ }^{1}$ S.O. Kostyukevych ${ }^{2}$ \\ ${ }^{1}$ Institute for information recording problems, NAS of Ukraine \\ 2, Shpak str., 03113 Kyiv, Ukraine, Phone: (044)-456-8389, fax: (044)-241-7233, e-mail: petrov@ipri.kiev.ua \\ ${ }^{2}$ V. Lashkaryov Institute of Semiconductor Physics, NAS of Ukraine, 41, prospect Nauky, 03028 Kyiv, Ukraine \\ e-mail: sekret@spie.org.ua
}

\begin{abstract}
Performed in this paper is the analysis of possibilities to create optical information carriers for long-term information storage. Adduced are the results of experimental investigations of properties inherent to optical carriers of the WORM type after 25-year storage. It has been shown that their micro-relief structure formed by using focused laser radiation on thin films of chalcogenide vitreous semiconductors had not been practically changed after storing them for the above mentioned period in non-heated areas.
\end{abstract}

Keywords: optical carrier, long-term storage, photo-thermal recording, chalcogenide vitreous semiconductor.

Manuscript received 28.05.09; accepted for publication 10.09.09; published online 30.10.09.

\section{Introduction}

Optical methods for information recording possess considerable potentialities to provide reliable long-term data storage. Realization of these opportunities is related with necessity to solve a plethora of interdependent tasks: choice of a carrier type and way to represent information, synthesis of registering media and technology for information recording on chosen substrates for optical carriers. For a long time, when developing the methods of optical recording, it was created, studied and used many types of optical carriers for various applications.

Disc carriers of the WORM type for photo-thermal recording were the first ones that found wide applications. Basic technical solutions that were used in creation of these carriers provided a high density and speed of information recording. But, most importantly, they ensured a high reliability of data storage [1 -3$]$. Application of plastic substrates in optical carriers allowed to essentially enhance processability in manufacturing disc optical carriers, but it resulted in essential shortening the guaranteed terms for data storage. A high reliability of data storage in optical carriers of the WORM type was provided due to the recording method based on photo-thermal destruction of absorbing layer (ablation recording). Almost for thirty years of investigations devoted to registering media for optical carriers with single-stage recording, there were offered many various types of registering media mainly based on organic dyes and semiconductor materials, multi-layer metal alloys. The performed investigations showed that the highest reliability of data storage is provided with the photo-thermal recording methods based on local destruction of the absorbing layer (i.e., ablation recording methods) as well as registering media with creation of thermo-stimulated tips caused by melting metallic films $[4,5]$. In recent years, there arise proposals in regard to using the optical carriers made in accord with the ablation recording method for long-term data storage with application of new light-absorbing materials [6]. Many attempts were made to estimate terms of data storage in WORM type optical carriers, and some accelerated tests to determine these terms [7] were performed. But it seems reasonable to analyze changes in optical carrier performances under conditions of long-term storage. In relation with it, the investigations of properties inherent to optical carriers, the information recording in which was realized 25 years ago using the ablation method.

\section{Substantiation for the choice of materials for registering media providing long-term data storage}

For a long time, to create registering media for WORM type optical carriers, tellurium alloys were widely used $[1-3]$. The choice of these materials was conditioned by the fact that thin films of tellurium alloys possess low thermal conductivity and low melting temperature. It provides high resolution of registering media based on tellurium vitreous alloys (minimum size of recorded pits is close to $0.3-0.5 \mu \mathrm{m}$ ) as well as the possibility to record information with low-powered semiconductor lasers. Performed experimental investigations showed 
that a dense oxide layer is created on the surface of tellurium glassy alloys.

In the process of creation of the WORM type optical disc carriers, the main attention was paid to the choice of materials for registering media capable to provide long-term storage of recorded data. Tens of glassy tellurium alloy compositions were studied to use them as registering media for optical disc carriers of the above type. In accord with preliminary calculations, to make registering media four-component eutectic alloy $\mathrm{Te}_{14} \mathrm{Sb}_{10} \mathrm{Se}_{61} \mathrm{Ge}_{15}$ [8] was chosen, which should provide long-term storage of recorded information. The distinction of using this alloy was in necessity to apply radiation with the wavelengths shorter than $550 \mathrm{~nm}$ to record information. Being aimed at performing the comparative investigations of optical carriers, there were made optical discs with registering media containing more than $60 \%$ of tellurium. Advantage of these carriers was that information recording in them could be realized with infra-red lasers. But the main reason to use just tellurium alloys instead of pure tellurium films was the necessity to increase the corrosion hardness of registering media. On the surface of tellurium alloys with $\mathrm{Sb}, \mathrm{Se}, \mathrm{Ge}$, there arise dense oxide films that reduce the speed of tellurium oxidation [9].

It was thought that the oxide film arising on the surface of vitreous tellurium layer will protect it against further oxygen penetration. The methods of accelerated aging (exposure of samples to increased humidity and temperature) showed that the speed of growth inherent to the thickness of this oxide layer is sharply reduced with increasing this thickness. The performed layer-by-layer Auger analysis of registering medium $\mathrm{Te}_{14} \mathrm{Sb}_{10} \mathrm{Se}_{61} \mathrm{Ge}_{15}$ manufactured 30 years ago showed that the oxygen content does not exceed 13 ат. \%, and oxygen is uniformly distributed along the thickness.

Depicted in Fig. 1 are the fragments of respective Auger spectra. In the initial state (Fig. 1a), at the oxygen background $475,490,511 \mathrm{eV}$ (the latter is the main peak) lines of $\mathrm{Sb}$ and $\mathrm{Te}$ are not registered. After etching the oxide film (Fig. 1b), Sb and Te peaks are developed (457 and $498 \mathrm{eV}$ ), and the oxygen content is reduced down to approximately 13 at.\%. In the case of tellurium, superposition of the main tellurium Auger peak with the oxygen one takes place. Observed there is the shift of the peak from the scale position 491 up to $498 \mathrm{eV}$.

It means that the oxygen-passivated film of multicomponent chalcogenides preserve its chemical composition, while the oxide film was easily etched by argon ion beam. It is noteworthy that oxygen distribution has a similar character both in unsealed registering media and in those covered with a protective polymer layer.

Availability of considerable oxygen amount in this registering media can be related with technological features of manufacturing the thin registering layer. When using vacuum deposition, there arises considerable amount of broken bonds in the structure of chalcogenide glass, which results in stage-by-stage accumulation of oxygen in this thin film.

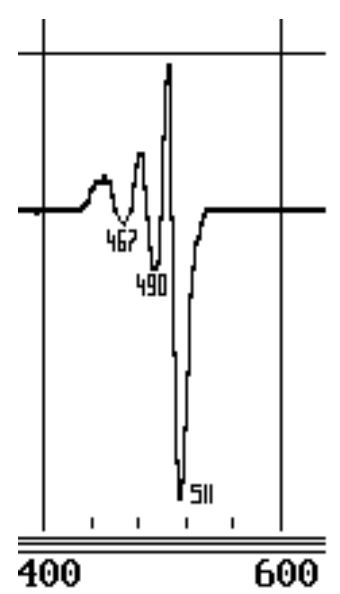

a)

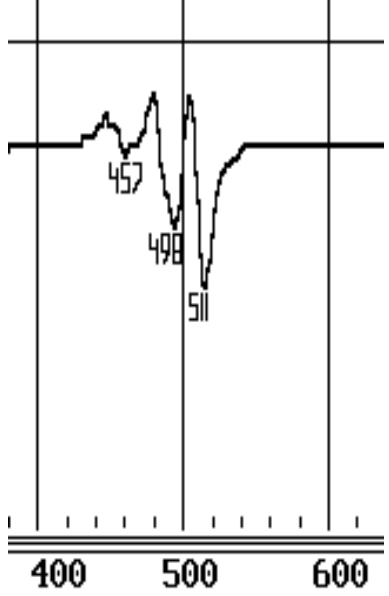

b)
Fig. 1. Distribution of oxygen in registering medium $\mathrm{Te}_{14} \mathrm{Sb}_{10} \mathrm{Se}_{61} \mathrm{Ge}_{15}$ before etching (a) and after etching with argon ion beam down to the depths $25-50 \mathrm{~nm}$ (b).

\section{Investigation results for optical carriers after long- term storage}

Recording information onto optical disc carriers with the registering layer $\mathrm{Te}_{14} \mathrm{Sb}_{10} \mathrm{Se}_{61} \mathrm{Ge}_{15}$ was realized using focused radiation of a solid-state laser with the wavelength $530 \mathrm{~nm}$. This record was made due to formation of pits in the absorbing chalcogenide layer. Information was registered on carriers of two types: first, we used optical disc carriers with open surface of the absorbing layer; second, this light-absorbing information layer was protected with a polymer film. In both cases, laser radiation was focused through transparent glass substrate of 3-mm thickness by using a special objective with the numeric aperture 0.65 . In the latter case, we joined two substrates of the optical carrier, the thickness of the polymer layer was close to $0.5 \mathrm{~mm}$. That provided independent recording process for each substrate of the optical carrier.

After long-term storage (25 years), these optical disc carriers kept mirror reflection, uniformity of coating layers within the area of data recording. The shape and sizes of pits appear to be slightly changed: the sizes are larger a little. Fig. 2 shows the look of the recording zone on the optical carrier after its 25-year storage.

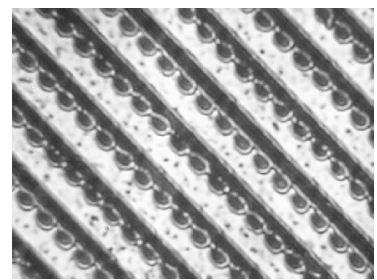

a)

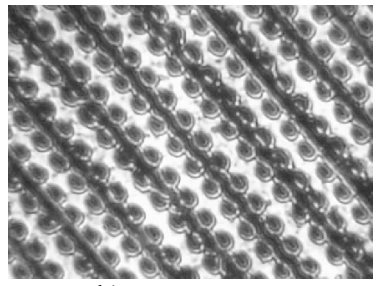

b)
Fig. 2. External view of the recording zone on the optical carrier: data recording is realized from one side of the guide track (a) and from both sides (b). 


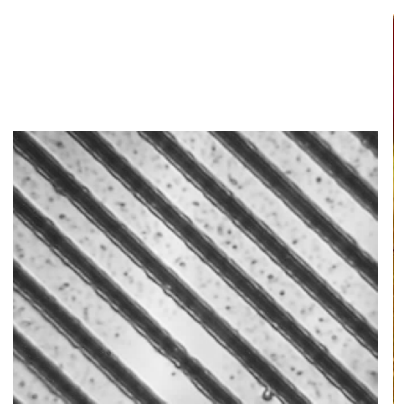

a)

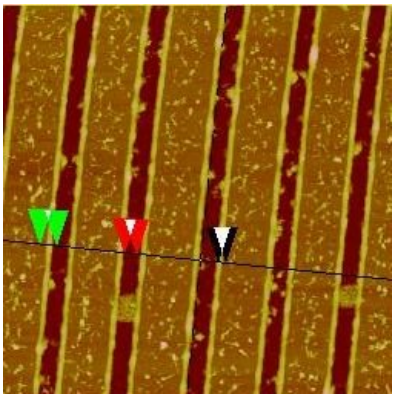

b)
Fig. 3. Guide tracks on the optical disc carrier with registering medium $\mathrm{Te}_{14} \mathrm{Sb}_{10} \mathrm{Se}_{61} \mathrm{Ge}_{15}$ (track pitch $1.6 \mu \mathrm{m}$ ). The images are obtained using: a) optical microscope; b) scanning tunnel microscope.

In the course of manufacturing the optical carrier, the surface of registering medium was processed with focused laser radiation to create concentric guide tracks. Information recording was realized on one or both sides of guide tracks. This way to form guide tracks is rather technological (it does not require any special preparation of the information carrier substrate, the tracks can be recorded using the facility for information recording). However, this way has essential deficiencies, namely:
- registering medium of the information carrier is separated by narrow strips, which can have a negative impact on the carrier storage term;

- availability of a bowl formed from material of registering medium along the guide tracks results in reduced density of information recording.

A higher density of recording can be reached in the case when guide tracks are formed in the substrate. It can be realized in glass substrates by using plasmochemical etching $[10,11]$. After long-term storage of the optical carrier with registering medium $\mathrm{Te}_{14} \mathrm{Sb}_{10} \mathrm{Se}_{61} \mathrm{Ge}_{15}$, guide tracks were kept unchanged, their sizes did not change within accuracy limits (Fig. 3).

Analogous changes in pit shapes were observed in the work [7]. The authors of this paper made the assumption that it is reasonable to use registering media based on chalcogenide semiconductors with nonreversible phase transitions for long-term data storage.

It is noteworthy that thin films with high tellurium content $\left(\mathrm{As}_{20} \mathrm{Se}_{20} \mathrm{Te}_{60}\right)$ become optically transparent after continuous storage in the same climatic conditions as those for the carriers where eutectic alloy $\mathrm{Te}_{14} \mathrm{Sb}_{10} \mathrm{Se}_{61} \mathrm{Ge}_{15}$ was used. On the surface of $\mathrm{As}_{20} \mathrm{Se}_{20} \mathrm{Te}_{60}$, one can visually observe information tracks by way of a diffraction grating. The shape of information tracks in the oxidized registering medium is depicted in Fig. 4.

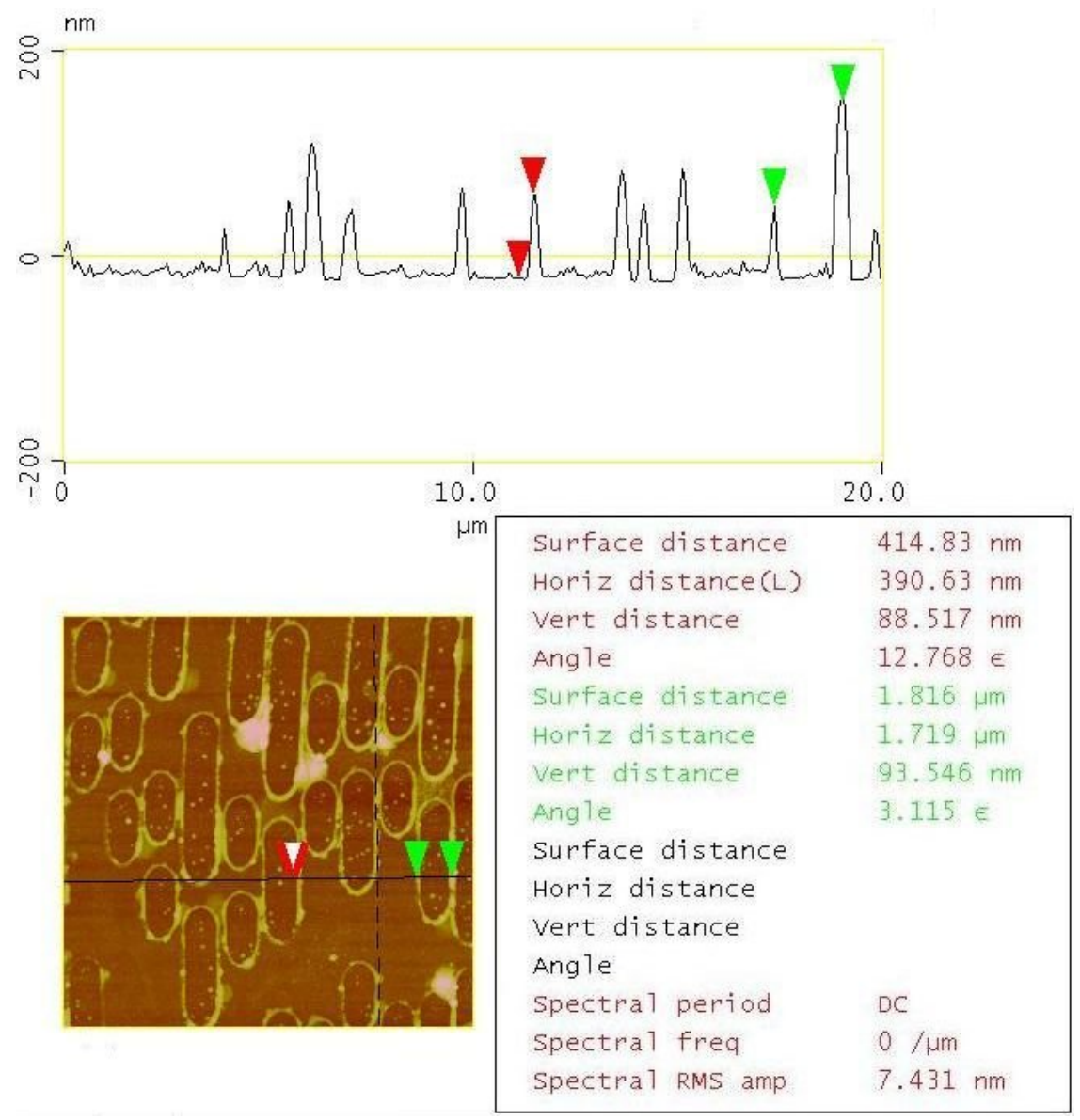

Fig. 4. Information tracks in the registering medium $\mathrm{As}_{20} \mathrm{Se}_{20} \mathrm{Te}_{60}$ after long-term storage. 


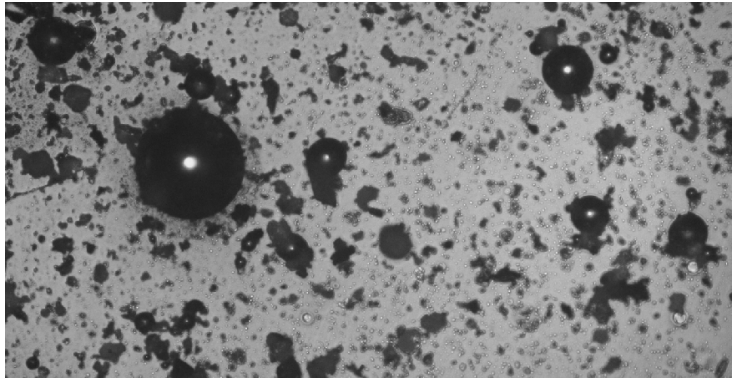

Fig. 5. The area of optical carrier registering medium with the tellurium content $40-50 \%$ after continuous storage.

Some samples of optical carriers with the tellurium content $40-50 \%$ in their registering media demonstrate surface crystallization, which results in losses of recorded information (Fig. 5).

As a whole, hopes to create optical disc carriers of the WORM type with glassy substrates based on vitreous chalcogenide semiconductors for long-term information storage are justified. However, our further investigations of properties inherent to optical carriers aimed at longterm storage have showed that optical carriers where micro-relief structure is formed in the glassy substrate based on highly stable material have considerably higher reliability. In optical carriers where data recording is realized in CD and DVD formats, to make these substrates one can use optically transparent silicate or borosilicate glasses as well as silica ones. To create Bluray carriers, beside the above mentioned materials one can use substrates made of monocrystalline ones.

\section{Conclusions}

1. Optical carriers with registering media made of vitreous chalcogenide semiconductors for information recording by local photo-thermal destruction of the absorbing layer with focused laser radiation keep their physical properties after long-term storage.

2. The shape and sizes of pits that code information show inessential changes: one can observe only a small increase in the pit sizes.

3. Observed is oxidation of components that comprise the composition of chalcogenide vitreous semiconductors used for manufacturing the registering medium of optical carriers.

\section{Acknowledgements}

The authors are very grateful to the specialists from the Institute for information recording problems, NAS of Ukraine, who assisted in optical information recording on these optical carriers and organized their long-term storage, as well as to the specialists from V. Lashkaryov Institute of Semiconductor Physics for the performed studying the pit profiles by using the scanning tunnel microscope.

\section{References}

1. Super-dense Optical Information Recording, V.V. Petrov, A.A. Kryuchin, S.M. Shanoilo, V.G. Kravets', I.O. Kossko, Ye.V. Belyak, A.S. Lapchuk, S.O. Kostyukevych. Kyiv, Institute for information recording, NAS of Ukraine, 2009. 282 pages. (in Ukrainian).

2. Optical-and-mechanical Storage Facility, V.V. Petrov, A.A. Kryuchin, A.P. Tokar' et al. Kiev, "Naukova dumka", 1992. 152 pages. (in Russian).

3. Non-organic photolithography, V.V. Petrov, A.A. Kryuchin, S.O. Kostyukevych, V.M. Rubish. Kyiv, Institute for Physics of Metals, NAS of Ukraine, 2007. 196 pages. (in Ukrainian).

4. W. Saffady. Optical Storage Technology, 1993: A state of Art Review. ISBN 0887368840. 1993.

5. Authors' certificate № 970449 USSR, Int. cl. index G 11 B 7/24. Carrier for optical recording. T.P. Doroshenko, A.A. Kryuchin, V.V. Petrov, G.Yu. Yudin. Applied 03.12.80. Published 30.10.82. Bulletin № 40.

6. Patent USA № 2008/0320205 A1. Int. cl. index G 06 F 12/00. Long-term digital data storage, Barry M. Lunt, Matthew R. Linford (USA), Brigham Young University (USA). Applied 20.06.2007. Published 25.12.2008.

7. Plasmon News. First Real-Time Tests Help Determine Security of Data Stored on WORD Media. 2004. www.plasmon.com.

8. Authors' certificate № 1340425 USSR, Int. cl. index G 11 B 7/00. Information carrier for optical storage facility. A.A. Kryuchin, V.V. Petrov, T.I. Sergiyenko, G.Yu. Yudin, Z.U. Borisova, M.D. Mikhailov, Yu.K. Lapin, A.V. Bogdanova. Applied 30.12.1985.

9. W. Lee, M. Chen, H. Weder, et al., Stability of thin Te and Te alloy films for optical data storage // Proc. SPIE 382, p. 282-289 (1983).

10. K. Sato, I. Okamoto, Y. Kitamoto, S. Ishida, Oblique ion nano-texturing technology for longitudinal recording media // Jpn. J. Appl. Phys. 46 (8A), p. 5139 -5142 (2007).

11. Rau Zhi-giang, Fang Hong-li, Fu Xin-ding, Chen Guo-ming, Fabricating master with reactive Ion Beam Etching Method // Chin. Phys. Lett. 15 (7), p. 495-497 (1998). 